2011-01-01

\title{
In-plane Sensitive Electronic Speckle Pattern Interferometer using a Diffractive Holographic Optical Element
}

Raghavendra Jallapuram

Technological University Dublin, mrutic@gmail.com

Con Healy

Technological University of Dublin, s0971014@sms.ed.ac.uk

Emilia Mihaylova

Technological University of Dublin, emilia.mihaylova@tudublin.ie

See next page for additional authors

Follow this and additional works at: https://arrow.tudublin.ie/cieoart

Part of the Optics Commons

\section{Recommended Citation}

Jallapuram, J. (2011) In-Plane Sensitive Electronic Speckle Pattern Interferometer Using a Diffractive Holographic Optical Element. American Journal of Physics Teachers, Vol. 74, no. 4, p.341-342. doi:10.1119/1.3533432

This Article is brought to you for free and open access by the Centre for Industrial and Engineering Optics at ARROW@TU Dublin. It has been accepted for inclusion in Articles by an authorized administrator of ARROW@TU

Dublin. For more information, please contact

arrow.admin@tudublin.ie, aisling.coyne@tudublin.ie, gerard.connolly@tudublin.ie.

Funder: Enterprise Ireland 


\section{Authors}

Raghavendra Jallapuram, Con Healy, Emilia Mihaylova, and Vincent Toal 


\title{
In-plane sensitive electronic speckle pattern interferometer using a diffractive holographic optical element
}

\author{
Raghavendra Jallapuram, Con Healy, Emilia Mihaylova, and Vincent Toal ${ }^{\text {a) }}$ \\ Centre for Industrial and Engineering Optics, Dublin Institute of Technology, Kevin Street, \\ Dublin 8, Ireland
}

(Received 19 July 2010; accepted 19 October 2010)

\begin{abstract}
We describe a student project in electronic speckle pattern interferometry. The project includes holographic recording of diffraction gratings in thick self-processing photopolymer layers made from off-the-shelf chemicals. The gratings are employed in a simple electronic speckle pattern interferometer to measure in-plane rotation. (C) 2011 American Association of Physics Teachers.

[DOI: $10.1119 / 1.3533432]$
\end{abstract}

\section{INTRODUCTION}

Electronic speckle pattern interferometry is a simple optical technique for measuring small displacements of optically rough surfaces. The technique is well known and easy to implement using simple optical components. Speckle pattern interferometry using a photographic method was first reported by Archbold et al. ${ }^{1}$ and further developed by Butters and Leendertz. ${ }^{2}$ Electronic speckle pattern interferometry has found many applications in nondestructive evaluation, ${ }^{3,4}$ mechanical stress analysis, ${ }^{5,6}$ and vibration analysis. ${ }^{7,8}$

In electronic speckle pattern interferometry the surface of an object is illuminated by laser light producing a speckled object beam which interferes with a coherent reference beam. The resulting speckle interferogram is imaged on a CCD camera, transferred to a computer, and saved in memory. When the surface is displaced, the speckle interferogram is changed due to the change in path length difference between the object and reference beams. This second interferogram is subtracted pixel by pixel from the first. The result is rectified and displayed as a set of bright and dark fringes, known as correlation fringes, which constitute a contour map of the displacement of the object surface. It is possible to view the fringe pattern changing in real time as the object surface displacement continues. Depending on the geometry, the interferometry system may be sensitive to inplane, out-of-plane displacement, or both.

Conventional in-plane sensitive systems usually involve the use of a beam splitter and/or mirrors to produce two collimated coherent beams, which illuminate the object at equal but opposite angles to the surface normal. The latter coincides with the optical axis of the camera lens. The beams should be of the same intensity to ensure maximum signalto-noise ratio in the fringe patterns. ${ }^{9}$

Holographic optical elements offer attractive alternatives to conventional optical elements in many applications ${ }^{10,11}$ and a diffractive holographic optical element (DHOE) can be used in a simple in-plane electronic speckle pattern interferometry system. The diffractive holographic optical element is placed close to the surface so that collimated obliquely incident light overlaps the first order diffracted light on the surface. Oblique incidence is necessary to allow the surface to be imaged on axis by the CCD camera through the diffractive holographic optical element [see Fig. 1(a)]. A thick element producing only one diffracted order along with the zero order is also necessary so that conventional gratings are not usually suitable. It is also easy to fabricate large aperture diffractive holographic optical elements in thick layers with the necessary 50\% efficiency, although bulk optical beam splitters with large apertures can be expensive.

\section{INTERPRETATION OF SPECKLE PATTERN INTERFEROMETRY FRINGES}

The in-plane sensitive electronic speckle pattern interferometer is shown in Fig. 1(a). Two collimated beams of mutually coherent light of wavelength $\lambda$ are incident on the surface of the object at equal and opposite angles, $\theta$. The intensity of light scattered from the surface along the normal is ${ }^{9}$

$$
I=I_{1}+I_{2}+2 \sqrt{I_{1} I_{2}} \cos \phi
$$

where $I_{1}$ and $I_{2}$ are the intensities of the two beams and $\phi$ is the phase difference between them.

When a point on the object undergoes a small in-plane displacement, $d$, in the plane of the illuminating beams, the phase difference between them is altered by $\Delta \phi$, which from Fig. 1(b) is

$$
\Delta \phi=\frac{4 \pi}{\lambda} d \sin \theta,
$$

and the intensity in Eq. (1) becomes

$$
I^{\prime}=I_{1}+I_{2}+2 \sqrt{I_{1} I_{2}} \cos (\phi+\Delta \phi) .
$$

The difference of Eqs. (1) and (3) gives

$$
\left|I-I^{\prime}\right|=\left|4 \sqrt{I_{1} I_{2}} \sin \left(\phi+\frac{\Delta \phi}{2}\right) \sin \left(\frac{\Delta \phi}{2}\right)\right|,
$$

which represents a fringe pattern having intensity maxima at $\Delta \phi=(2 p+1) \pi$, with $p$ as an integer, and minima at $\Delta \phi$ $=2 p \pi$, the fringes being modulated by speckle.

If $d$ is spatially dependent, the phase change gives rise to bright and dark fringes according to Eq. (4). From Eq. (2) the in-plane displacement undergone by a given point is

$$
d=\frac{n \lambda}{2 \sin \theta},
$$

where $n$ is the number of fringes passing through the point during the displacement $d$.

The simplest, spatially dependent, in-plane displacement is the rotation of a flat circular disk in the $x-y$ plane as shown in Fig. 1(c). The wave vectors of the illuminating beams lie in the $x-z$ plane. If the disk rotates through an angle $\delta \alpha$, a point with polar coordinates $r, \alpha$ is displaced by $r \delta \alpha \cos \alpha$ in 


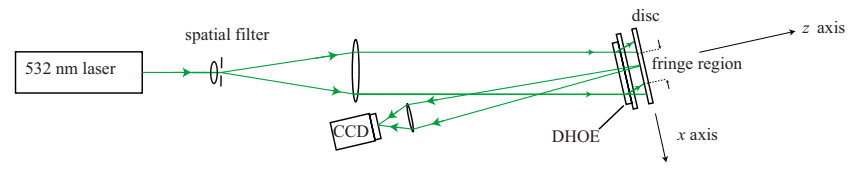

(a)

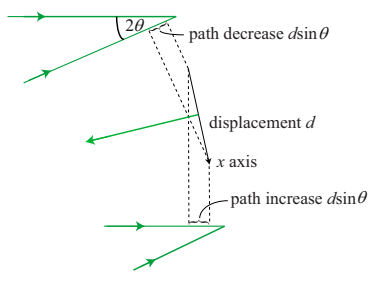

(b)

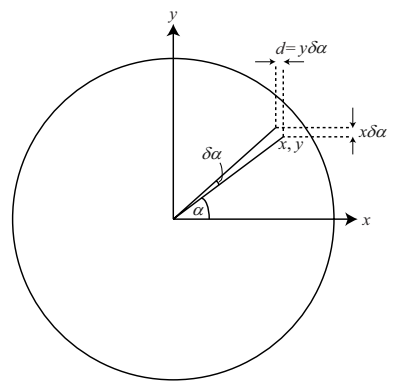

(c)
Fig. 1. (a) In-plane electronic speckle pattern interferometer using a DHOE; (b) path change due to displacement; and (c) in-plane rotation. All points on a horizontal straight line are equally displaced.

the $y$ direction and by $r \delta \alpha \sin \alpha=y \delta \alpha$ in the $x$ direction [see Fig. 1(c)]. The phase change at the point $x, y$ depends only on $y \delta \alpha$ and the speckle pattern fringes lying parallel to the $x$ direction are observed on the display. From Eq. (5) the angle of rotation, $\delta \alpha$, is given by

$$
\delta \alpha=\frac{n \lambda}{2 L \sin \theta},
$$

where $n$ is the number of fringes seen in the vertical field of view, $L$.

\section{EXPERIMENT}

To record the diffractive holographic optical elements, we used a self-processing photopolymer developed at the Centre for Industrial and Engineering Optics, Dublin Institute of Technology ${ }^{12}$ for holographic applications. The photopolymer was prepared using polyvinyl alcohol (PVA) (80\% hydrolysed, 9000-10000 g/mol molecular weight), acrylamide, $N N^{\prime}$-methylene bisacrylamide, triethanolamine, and erythrosine B photosensitive dye. All the chemicals were from Sigma Aldrich and used as obtained.

A $10 \% w / v$ aqueous solution of PVA was prepared by dissolving $10 \mathrm{~g}$ of PVA, a little at a time, in $100 \mathrm{ml}$ of distilled water at approximately $80{ }^{\circ} \mathrm{C}$ with constant stirring until completely dissolved. The solution was then allowed to cool to room temperature. A stock solution of photosensitive dye was prepared by dissolving $0.11 \mathrm{~g}$ of erythrosine $\mathrm{B}$ dye in $100 \mathrm{ml}$ of distilled water. This dye has its absorption maximum at $532 \mathrm{~nm}$, matching the recording laser wavelength. Methylene blue dye in the same concentration may be used instead to sensitize the photopolymer for diffractive holographic optical element recording at $633 \mathrm{~nm}$ with a $\mathrm{He}-\mathrm{Ne}$ laser.

The photopolymer solution was prepared by adding $0.6 \mathrm{~g}$ of acrylamide and $0.2 \mathrm{~g}$ of $N N^{\prime}$-methylene bisacrylamide to $2 \mathrm{ml}$ of triethanolamine in a fume hood. $18 \mathrm{ml}$ of $10 \% \mathrm{w} / \mathrm{v}$ aqueous stock solution of PVA was then added. The solution was stirred until the monomers were completely dissolved. 4 $\mathrm{ml}$ of erythrosine B stock solution was added and stirred until a homogenous solution was obtained. $2 \mathrm{ml}$ of photo-

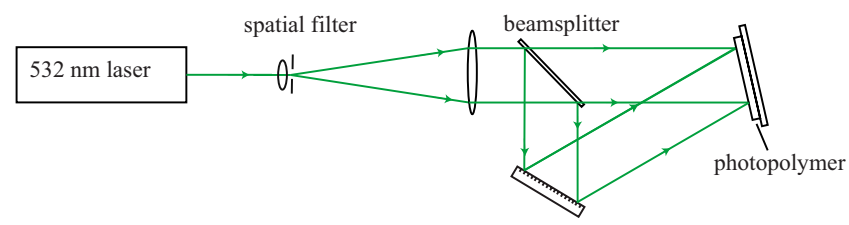

Fig. 2. Diffractive holographic optical element recording.

polymer solution was deposited on a $63 \times 63 \mathrm{~mm}^{2}$ clear glass leveled plate and allowed to dry at ambient temperature, which normally takes about $12 \mathrm{~h}$. The thickness of the dry layer, measured using a white light surface profilometer, was $100 \mu \mathrm{m}$. A micrometer gauge can also be used, with care, for thickness measurements.

Figure 2 shows the optical setup used to record the diffractive holographic optical elements. The components were mounted on a mechanically isolated optical table. An optical breadboard supported on partially inflated inner tubes (motor scooter tires) serves very well. The exposure was adjusted to be around $50 \mathrm{~mJ} \mathrm{~cm}^{-2}$ until zero and first order diffracted beams of equal intensity were obtained from the recorded gratings. A suitable angle, $2 \theta$, between the recording beams is obtained from Eq. (6). For example, an angular rotation of the disk by $1.0 \mathrm{mrad}$ results in ten electronic speckle pattern interferometry fringes in a field of view of $1 \mathrm{~cm}$ when using a diffractive holographic optical element recorded at $532 \mathrm{~nm}$ with an angle of $30^{\circ}$ between the beams in Fig. 2 .

The circular disk was rotated in plane by a motor driven micrometer. The angle of rotation was independently measured by the displacement of a He-Ne laser reflected onto the wall of the laboratory from a small plane mirror attached to the rim of the disk. The field of view was measured on the display by placing a ruler against the disk.

\section{RESULTS AND DISCUSSION}

Figure 3 shows some of the electronic speckle pattern interferometry fringe patterns we obtained. The fringe contrast was very good initially, although it became difficult to count fringes with complete confidence when about 15 had accu-

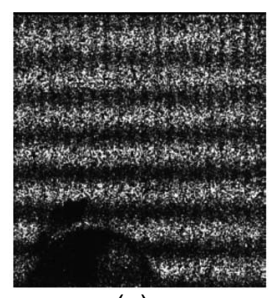

(a)

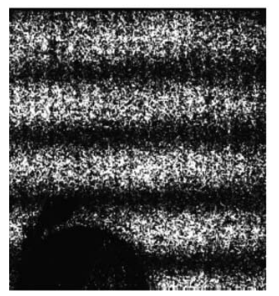

(c)

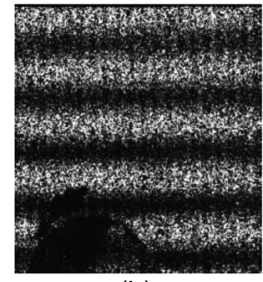

(b)

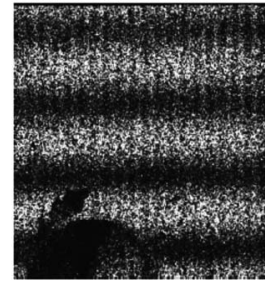

(d)
Fig. 3. Electronic speckle pattern interferometry fringe patterns for in-plane rotations of (a) 1.1, (b) 0.80 , (c) 0.69 , and (d) $0.58 \mathrm{mrad}$. The field of view is $9 \mathrm{~mm}$. The dark area is a bolt securing the disk to the rotator. 


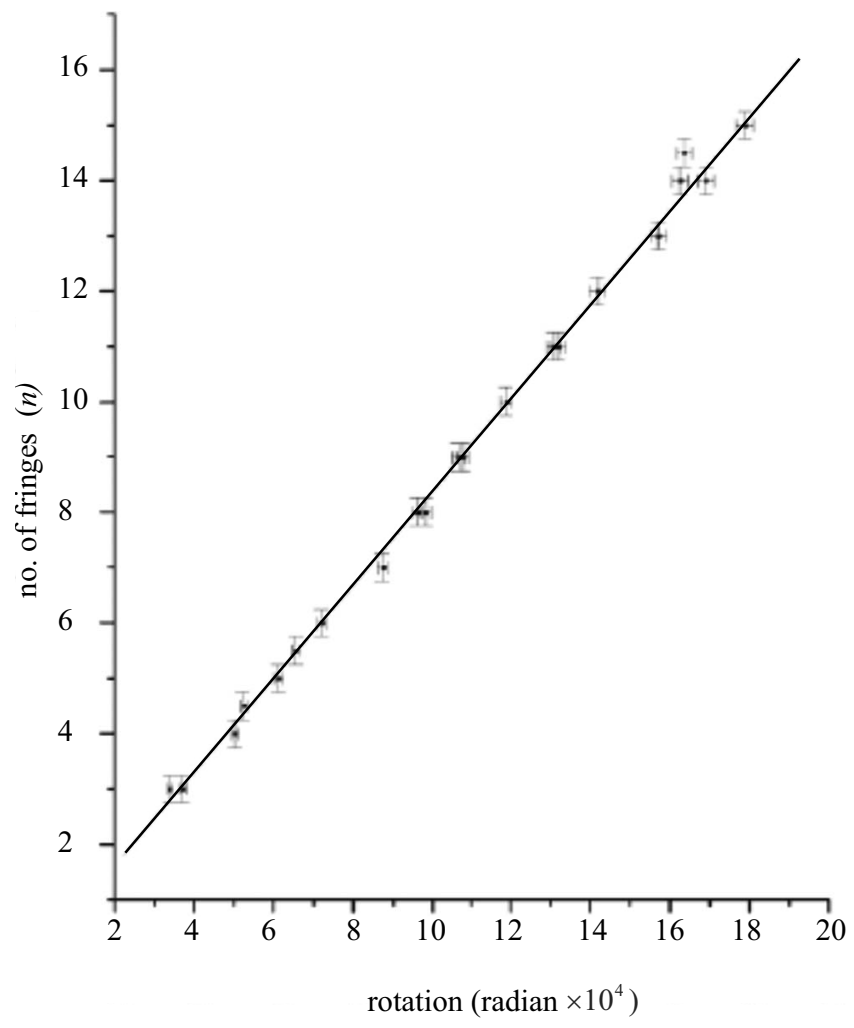

Fig. 4. Number of electronic speckle pattern interferometry fringes in the field of view versus angle of rotation.

mulated in the image because decorrelation of the speckle interferograms, $I$ and $I^{\prime}$, occurs as speckles move away from the CCD element at which they were initially located. ${ }^{9}$ The number of fringes in the field of view is plotted against the angle of rotation in Fig. 4. There are two sources of experimental errors. One is the accuracy of fringe counts, which depends on the total number and was here to be one quarter of a fringe. The other source is in the measurement of the angle of diffraction of the diffractive holographic optical element, obtained here by measurements of the triangle defined by the point of incidence of an unexpanded laser on the diffractive holographic optical element and the points of incidence of the zero order and diffracted beams on the laboratory wall.

\section{CONCLUSION}

An in-plane sensitive electronic speckle pattern interferometer was implemented using a diffractive holographic optical element that was holographically recorded in a photopolymer. Fringes of good contrast were obtained and used in the measurement of in-plane rotation of a disk. A diffractive holographic optical element can be easily substituted for one with the grating spacing suited to the estimated magnitude of the rotation to be measured. The project can be extended to the measurement of in-plane strains.

\section{ACKNOWLEDGMENTS}

One of the authors (C.H.) acknowledges financial support from the Science Foundation Ireland UREKA undergraduate research experience program. The authors thank the reviewers for their helpful suggestions.

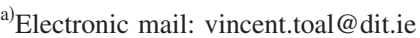

${ }^{1}$ E. Archbold, J. M. Burch, A. E. Ennos, and P. A. Taylor, "Visual observation of surface vibration nodal patterns," Nature (London) 222, 263265 (1969).

${ }^{2}$ J. N. Butters and J. A. Leendertz, "Speckle pattern and holographic techniques in engineering metrology," Opt. Laser Technol. 3, 26-30 (1971).

${ }^{3}$ C. Joenathan, B. Franze, P. Haible, and H. J. Tiziani, "Nondestructive testing using temporal phase evaluation in speckle interferometry," Exp. Mech. 40, 106-111 (2000).

${ }^{4}$ E. Mihaylova, B. Potelon, S. Reddy, V. Toal, and C. Smith, "Mechanical characterization of unplasticised polyvinylchloride thick pipes using electronic speckle pattern interferometry," Opt. Lasers Eng. 41, 889-900 (2004).

${ }^{5}$ Dong-Won Kim, Nak-Kyu Lee, Kyoung-Hoan Na, and Dongil Kwon, "Analysis by speckle interferometry of the dependency of yield stress on residual stress," Opt. Lasers Eng. 43, 221-232 (2005).

${ }^{6}$ A. A. Maas and H. A. Vrooman, "In-plane strain measurement by digital phase shifting interferometry," Proc. SPIE 1162, 248-256 (1990).

${ }^{7}$ D. Borza and I. Nistea, "Application of digital speckle interferometry for vibration analysis of a statically loaded vehicle tyre," Acoustics '08, 5453-5456 (2008).

${ }^{8}$ E. Mihaylova, B. Viswanath, R. Jallapuram, and V. Toal, "Whole field out-of-plane vibration analysis with a HOE-based ESPI system," Proc. SPIE 7098, 70980M (2008).

${ }^{9}$ R. Jones and C. Wykes, Holographic and Speckle Interferometry, 2nd ed. (Cambridge U. P., Cambridge, 1989).

${ }^{10}$ J. E. Ludman, "Holographic solar concentrator," Appl. Opt. 21, 30573058 (1982)

${ }^{11}$ E. A. Ash, E. Seaford, O. Soares, and K. S. Pennington, "Holographic coupler for integrated optics," Appl. Phys. Lett. 24, 207-208 (1974).

${ }^{12}$ I. Naydenova, R. Jallapuram, S. Martin, R. Howard, and V. Toal, "Investigations of the diffusion processes in self-processing acrylamide-based photopolymer system," Appl. Opt. 43, 2900-2905 (2004). 